\title{
BDVCM about the Steel Box Girder Reliability Prediction Based on Monitoring Extreme Stresses
}

\author{
Yuefei Liu, ${ }^{1}$ Xueping Fan $\mathbb{D}^{1},{ }^{1}$ Guanghong Yang, ${ }^{2}$ Zhipeng Shang, ${ }^{2}$ and Xiaoxiong Zhao ${ }^{2}$ \\ ${ }^{1}$ Key Laboratory of Mechanics on Disaster and Environment in Western China (Lanzhou University), \\ The Ministry of Education of China, School of Civil Engineering and Mechanics, Lanzhou University, Lanzhou 730000, China \\ ${ }^{2}$ School of Civil Engineering and Mechanics, Lanzhou University, Lanzhou, 730000, China \\ Correspondence should be addressed to Xueping Fan; fanxp@lzu.edu.cn
}

Received 16 July 2020; Revised 28 December 2020; Accepted 3 February 2021; Published 20 February 2021

Academic Editor: Pier Paolo Rossi

Copyright (C) 2021 Yuefei Liu et al. This is an open access article distributed under the Creative Commons Attribution License, which permits unrestricted use, distribution, and reproduction in any medium, provided the original work is properly cited.

To reasonably predict the steel box girder reliability considering the dynamic dependence among the performance functions corresponding to the failure modes of the multiple monitoring points, this paper firstly adopts the dynamic monitoring extreme stresses of the multiple control points to build the Bayesian Dynamic Vine Copula Model (BDVCM) taking into account the dynamic dependence of the multiple monitoring variables through combining the vine copula technique with Bayesian Dynamic Linear Models (BDLM); secondly, with first-order second-moment method and the built BDVCM, the steel box girder reliability, taking into account dynamic dependence among the performance functions corresponding to the failure modes of the multiple monitoring points, is predicted; finally, the monitoring data from the five sections of an existing steel box girder were provided to illustrate the proposed model and approach. The analytical results illustrated that the predicted results, without considering the dynamic nonlinear dependence among the failure modes of the multiple monitoring points, are conservative.

\section{Introduction}

Structural Health Monitoring (SHM) systems have accumulated a large amount of monitored data in the long-term service periods. How to reasonably predict bridge system reliability with these data has become one of the key scientific problems in SHM field. Because of the same input loads, the different monitoring data of the multiple monitoring points from bridge system show the dependence, randomness, and so on. Further, dynamic dependence among the performance functions corresponding to the multiple failure modes of the monitoring points can be shown. Therefore, based on the dependent monitoring data, the dependence models of the multiple failure modes can be presented; further, it is more reasonable to dynamically predict the bridge girder reliability.

Until now, some studies about the dynamic reliability prediction of bridge systems, taking into account the dynamic dependence among the multiple failure modes, have been done based on copula functions; for example, a copula-based method is presented to investigate the impact of copulas characterizing the dependence structures among the random variables for modeling bivariate distributions, respectively, on time-independent and time-dependent system reliability under incomplete probability information $[1,2]$; a new model named BDGCM for characterizing the dynamic dependence between only two monitoring points is firstly built [3], and, based on it, the bridge system reliability is dynamically predicted, where correlation coefficients are not accurately computed and the built BDLM does not take the nonlinear dependence between two variables into account; copulafunctions-based dynamic dependence model between only two monitoring points of arch bridge girder is given [4], where the correlation coefficients are approximately obtained and the nonlinear correlation between two monitoring variables is not taken into account in the built BDLM; Gaussian copula-Bayesian Dynamic Linear Model-based dynamic reliability prediction for Yitong River Bridge (a 
flying swallow profiled concrete-filled steel tube arch bridge) taking into account the time-variant dependence between the performance functions of only one pair of failure modes is made $[5,6]$, where the correlation coefficients are not accurately solved and the built BDLM also does not consider the nonlinear dependence between a pair of variables. The above research studies show that the existing dynamic reliability prediction methods of bridge structures considered timevariant nonlinear dependence between one pair of performance functions, and the correlation coefficients are not accurately solved. Meanwhile, actual bridge systems commonly have multiple control monitoring points. Therefore, building more accurate dynamic nonlinear dependence models among the multiple failure modes corresponding to the control monitoring points and further predicting the dynamic reliability of bridge systems should be further studied and become the aim of the present research.

In view of the above problems, this study adopts the steel box girder as the research object, takes a newly built Bayesian Dynamic Vine Copula Model (BDVCM) to characterize the dynamic nonlinear correlations among the performance functions of the failure modes at multiple control monitoring points of the steel box girder based on BHM data, and further dynamically predicts the steel box girder reliability. In this paper, monitoring extreme data is "the everyday extreme stress data." For each section, the control monitoring point is "the monitoring point where the absolute value of the monitoring stress is maximal"; each failure mode refers to the fact that "the monitoring extreme stress is bigger than the allowable stress." The detailed contents are as follows: firstly, based on monitoring extreme stress data, the BDVCM about the multiple monitoring variables is built, which can characterize the dynamic dependence among the monitoring variables; secondly, with first-order second-moment (FOSM) method and the built BDVCM, the dynamic reliability prediction method of the steel box girder, considering dynamic nonlinear correlations among the performance functions for multiple failure modes corresponding to the multiple control monitoring points, is given; thirdly, the monitoring extreme stress data of a steel box girder are provided to illustrate the feasibility and application of the proposed model and method; finally, some valuable conclusions are summarized.

\section{BDVCM of Multiple Monitoring Variables}

This section firstly adopts the dynamic monitoring extreme stress data to establish the corresponding Bayesian Dynamic Linear Models (BDLM) about the multiple monitoring variables of the steel box girder sections, and the BDLM can dynamically predict the extreme data of monitoring variables; then, the vine copula model, characterizing the nonlinear correlation among multiple monitoring variable about the steel box girder sections, is built; finally, the Bayesian Dynamic Vine Copula Model (BDVCM) about multiple monitoring variables is given through combining the built BDLM with vine copula. The detailed contents are as follows.
2.1. BDLM of Monitoring Variables and the Corresponding Recursive Processes. Bayesian Dynamic Linear Models (BDLM) include Dynamic Linear Models (DLM) and the corresponding recursive processes based on Bayes method. The detailed contents are as follows.

2.1.1. Construction of DLM. The DLM includes the monitoring equation, state equation, and initial state information, where the state equation shows changes of the system with time and reflects inner dynamic changes of the system and random disturbances; the monitoring equation expresses the relationship between the monitoring data and the current state parameters of the system; the initial state information is the probability distribution information about the initial state.

Suppose that all the dynamic state variables $\left\{\theta_{t, i}\right.$, $i \in\{1,2, \ldots, n\}\}$ follow normal distribution, are all Markov chains, and are internally independent and mutually independent between each other. The state error $\omega_{i, t}$ and the monitoring error $v_{i, t}$ all follow normal distribution and are internally independent and mutually independent between each other. DLM is defined in the following form.

Monitoring equation:

$$
y_{i, t}=\theta_{i, t}+v_{i, t}, v_{i, t} \sim \mathrm{N}\left(0, V_{i, t}\right) .
$$

State equation:

$$
\theta_{i, t}=\theta_{i, t-1}+\omega_{i, t}, \omega_{i, t} \sim \mathrm{N}\left(0, W_{i, t}\right) .
$$

Initial state information:

$$
\left(\theta_{i, t-1} \mid D_{i, t-1}\right) \sim \mathrm{N}\left(m_{i, t-1}, C_{i, t-1}\right)
$$

where $y_{i, t}, v_{i, t}$, and $\omega_{i, t}$ are, respectively, the monitoring extreme stress data, monitoring error, and state error about the DLM of the $i^{\text {th }}$ monitoring variable at time $t ; \mathrm{N}(\cdot)$ is normal distribution function; $V_{i, t}$ is a variance; $W_{i, t}$ is an evolution variance indicating the model recurrence uncertainty from time $t$ - 1 to time $t ; \mathbf{D}_{i, t-1}$ is the information set of the $i^{\text {th }}$ monitoring variable at and before time $t-1$, and $\mathbf{D}_{i, t}=$ $\left\{y_{i, t}, \mathbf{D}_{i, t-1}\right\}$ is the information set of the DLM about the $i^{\text {th }}$ monitoring variable at and before time $t$, which includes $m_{i, t}$ (mean) and $C_{i, t}$ (variance). It is assumed that error sequences $v_{i, t}$ and $\omega_{i, t}$ are internally independent, mutually independent, and independent of $\theta_{i, t}$.

2.1.2. Bayesian Probability Recursive Processes of DLM. In this section, the Bayesian probability recursive processes of DLM are provided, which are as follows:

(1) The a posteriori probability density function (PDF) of the state at time $t-1$ :

$$
\left(\theta_{i, t-1} \mid \mathbf{D}_{i, t-1}\right) \sim \mathrm{N}\left(m_{i, t-1}, C_{i, t-1}\right) \text {. }
$$

(2) The a priori distribution of the state at time $t$ :

$$
\left(\theta_{i, t} \mid \mathbf{D}_{i, t-1}\right) \sim \mathrm{N}\left(a_{i, t}, R_{i, t}\right) \text {. }
$$


(3) One-step prediction distribution of monitoring variable at time $t$ :

$$
\left(y_{i, t} \mid \mathbf{D}_{i, t-1}\right) \sim \mathrm{N}\left(\mu_{i, t}, \sigma_{i, t}^{2}\right) .
$$

where $\quad \mu_{i, t}=\mathrm{E}\left(y_{i, t} \mid \mathbf{D}_{i, t-1}\right)=a_{i, t}, \quad Q_{i, t}=\sigma_{i, t}^{2}=$ $\operatorname{var}\left(y_{i, t} \mid \mathbf{D}_{i, t-1}\right)=R_{i, t}+V_{i, t}$, and $\left(Q_{i, t}\right)^{-1}$ is the prediction precision of BDLM.

According to the definition of Highest Posterior Density (HPD) region [7], the one-step prediction interval of the monitoring variable with a $95 \%$ guarantee rate at time $t$ is

$$
\left[\mu_{i, t}-1.645 \sqrt{\sigma_{i, t}^{2}}, \mu_{i, t}+1.645 \sqrt{\sigma_{i, t}^{2}}\right]
$$

where $\mu_{i, t}-1.645 \sqrt{\sigma_{i, t}^{2}}$ is the predicted lower limit data and $\mu_{i, t}+1.645 \sqrt{\sigma_{i, t}^{2}}$ is the predicted upper limit data.

(4) The a posteriori distribution of the state at time $t$ :

$$
\left(\theta_{i, t} \mid \mathbf{D}_{i, t}\right) \sim \mathrm{N}\left(m_{i, t}, C_{i, t}\right),
$$

where $m_{i, t}=\mathrm{E}\left(\theta_{i, t} \mid \mathbf{D}_{i, t}\right)=a_{i, t}+A_{i, t} e_{i, t} ; C_{i, t}=\operatorname{var}\left(\theta_{i, t} \mid \mathbf{D}_{i, t}\right)=$ $R_{i, t}-A_{i, t} A_{i, t}^{T} \sigma_{i, t}^{2} ; A_{i, t}=R_{i, t}\left(\sigma_{i, t}^{2}\right)^{-1} ; e_{i, t}=y_{i, t}-f_{i, t} ; A_{i, t}$ is the adaptive coefficient.

\subsubsection{Determination of the Main Parameters about BDLM.} In this paper, the updating time interval of BDLM is one day. The main probability distribution parameters about BDLM include $V_{i, t}, W_{i, t}, m_{i, t-1}$, and $C_{i, t-1}$.

The monitoring extreme data at and before time $t$ are smoothly processed with cubical smoothing algorithm with five-point approximation [3, 5-7], and the processed data can be approximately taken as the initial state data [3, 7]. Through carrying out parameter estimation for the initial state data, $m_{i, t-1}$ and $C_{i, t-1}$ can be obtained. The variance $V_{i, t}$ of the monitoring errors can be approximately solved with the difference between monitoring extreme data and the initial state data; the discount factor $\delta_{i}$ is considered as an aid to choosing $W_{i, t}$. In the proposed BDLM, the predicting behavior is usually rapidly achieved. It is convenient and natural to adopt a constant rate of increase of uncertainty or decay of information. Thus, for a given discount factor, equation (9) is chosen to solve the variance $W_{i, t}$ of the state error.

$$
W_{i, t+1}=-C_{i, t}+\frac{C_{i, t}}{\delta}
$$

where $\delta_{i}$ is the discount factor and $\delta_{i}=0.98$ according to the research experience of the authors $[3,7]$.
2.2. Vine Copula Model of Five-Dimensional Monitoring Variables. In this study, the bridge girder has multiple monitoring points which are corresponding to multiple output variables. Because of the same input vehicle loads, environmental loads, and so on, there exists nonlinear correlation between these output variables. In this section, the vine copula model considering nonlinear correlation of the five output variables is built with pair-copula theory and bivariate Gaussian copula theory.

2.2.1. Pair-Copula Theory. Pair-copula construction module based joint probability distribution function of multivariate random variables is proposed by Bedford $[8,9]$. The paircopula construction module only considers the nonlinear correlation of two random variables.

Assume that there is a five-dimensional random variable $X=\left(x_{1}, x_{2}, \ldots, x_{5}\right)$, and, according to the conditional probability density function theory [10], copula model based joint probability density function $f\left(x_{1}, x_{2}, \ldots, x_{5}\right)$ can be written as

$$
f\left(x_{1}, x_{2}, \ldots, x_{5}\right)=c\left(F_{1}\left(x_{1}\right), F_{2}\left(x_{2}\right), \ldots, F_{5}\left(x_{5}\right)\right) \prod_{i=1}^{5} f_{i}\left(x_{i}\right)
$$

where $c$ is copula density function and $F_{i}\left(x_{i}\right)$ and $f_{i}\left(x_{i}\right)$ are, respectively, cumulative distribution function (CDF) and probability density function (PDF) of $x_{i}$.

With equation (10), the bivariate joint PDF can be obtained with

$$
f\left(x_{i}, x_{j}\right)=c_{i j}\left(F_{i}\left(x_{i}\right), F_{j}\left(x_{j}\right)\right) f_{i}\left(x_{i}\right) f_{j}\left(x_{j}\right),
$$

and, further, conditional PDF can be obtained; namely,

$$
f\left(x_{i} \mid x_{j}\right)=\frac{f\left(x_{i}, x_{j}\right)}{f_{2}\left(x_{j}\right)}=c_{i j}\left(F_{i}\left(x_{i}\right), F_{j}\left(x_{j}\right)\right) f_{i}\left(x_{i}\right),
$$

and, with equation (12), conditional PDF about each variable $x$ of five-dimensional random variables can be reached; namely,

$$
f(x \mid u)=c_{x u_{i} \mid u_{-i}}\left(F\left(x \mid u_{-i}\right), F\left(u_{i} \mid u_{-i}\right)\right) f\left(x \mid u_{-i}\right),
$$

where $u_{i}$ represents the $i^{\text {th }}$ variable of five-dimensional random variables and $u_{-i}$ represents the four-dimensional random variables obtained through removing the $i^{\text {th }}$ variable from five-dimensional random variables.

2.2.2. Bivariate Gaussian Copula Model. This section adopts the bivariate Gaussian copula model $[6,11]$ to build the nonlinear correlation model between two random variables. The CDF and PDF about the bivariate Gaussian copula model are, respectively, 


$$
\begin{aligned}
& C\left(u_{1}, u_{2} ; \rho\right)=\Phi_{G}\left(\Phi^{-1}\left(u_{1}\right), \Phi^{-1}\left(u_{2}\right) ; \rho\right)=\int_{-\infty}^{\Phi^{-1}\left(u_{1}\right)} \int_{-\infty}^{\Phi^{-1}\left(u_{2}\right)} \frac{1}{2 \pi\left(1-\rho^{2}\right)(1 / 2)} \exp \left(\frac{-\left(r^{2}-2 \rho r s+s^{2}\right)}{2\left(1-\rho^{2}\right)}\right) \mathrm{d} r \mathrm{~d} s \\
& c\left(u_{1}, u_{2} ; \rho\right)=\frac{1}{\sqrt{1-\rho^{2}}} \exp \left(\frac{\Phi^{-1}\left(u_{1}\right)^{2}+\Phi^{-1}\left(u_{2}\right)^{2}-2 \rho \Phi^{-1}\left(u_{1}\right) \Phi^{-1}\left(u_{2}\right)}{2\left(1-\rho^{2}\right)}\right) \exp \left(-\frac{\Phi^{-1}\left(u_{1}\right)^{2} \Phi^{-1}\left(u_{2}\right)^{2}}{2}\right) .
\end{aligned}
$$

where $u_{i}=F_{i}\left(x_{i}\right), i=1,2, \ldots, 5, F_{i}\left(x_{i}\right)$ is the marginal CDF of $x_{i} ; \Phi$ is standard normal distribution function; and $\rho$ restricted to the interval $[-1,1]$ is the relevant parameter of Gaussian copula function.

In this paper, the relevant parameter $\rho$ of bivariate Gaussian copula function is determined by the Pearson linear correlation coefficient [4-6].

Suppose that Pearson linear correlation coefficient of random variables $x_{i}$ and $x_{j}$ is $\rho(i, j)$. The relevant parameter $\rho$ of bivariate Gaussian copula function can be obtained with the following equation:

$$
\begin{aligned}
\rho & =\rho\left(\Phi^{-1}\left(u_{i}\right), \Phi^{-1}\left(u_{j}\right)\right)=\rho\left(\Phi^{-1}\left(F_{i}\left(x_{i}\right)\right), \Phi^{-1}\left(F_{j}\left(x_{j}\right)\right)\right) \\
& =\rho\left(x_{i}, x_{j}\right)=\rho(i, j) .
\end{aligned}
$$

\subsubsection{Vine Copula Model of Five-Dimensional Random} Variables. This section adopts multiple pair-copula models to construct the multivariate joint PDF. From the definition of vine structures $[8,9]$, as can be known that each vine consists of multiple trees, each tree contains multiple nodes, the line connecting two nodes is termed as edge, and different edges are independent of each other. Different vine structures have different properties, and the widely used ones are regular vine structures $[8,9]$, which include many types. The widely applied regular vine structure types are $\mathrm{C}$-vine and $\mathrm{D}$-vine. This section takes five-dimensional random variables as the example to build C-vine structure and D-vine structure, respectively, as shown in Figures 1 and 2. Figure 1 shows that $\mathrm{C}$-vine structure of five-dimensional random variables contains four trees, and every tree has one main node that is connected to the other nodes. Each line (edge) connecting main node to the other node is corresponding to one pair-copula model, and different lines (edges) are independent of each other. Figure 2 shows that $\mathrm{D}$-vine structure of five-dimensional random variables also has four trees and ten edges (lines), and each edge is corresponding to one pair-copula model. Through Figure 1, $\mathrm{C}$-vine is composed of ten pair-copula models, and any two pair-copula models are independent. Meanwhile, in Figure 2, D-vine is also composed of ten pair-copula models, and any two pair-copula models are also independent.

2.3. BDVCM of Five-Dimensional Monitoring Variables. In this section, the Bayesian Dynamic Vine Copula Model (BDVCM) is a fusion of BDLM and vine copula models. The multivariate joint distribution function is decomposed into a series of BDVCM about any two monitoring variables; further, the BDVCM of multiple monitoring variables can be obtained through connecting the BDVCM of any two monitoring variables with vine structure.

With equation (6) and [4-6], the dynamic Pearson linear correlation coefficient between any two monitoring variables $\left(x_{i}, x_{j}\right)$ can be expressed with

$$
\rho_{t}(i, j) \approx \frac{\operatorname{cov}\left(D_{i, t-1}, D_{j, t-1}\right)}{\sigma_{i, t} \sigma_{j, t}},
$$

and, with equations (15) and (16), the dynamic relevant parameter $\rho_{t}$ of bivariate Gaussian copula function can be obtained with

$$
\rho_{t}=\rho_{t}(1,2)
$$

The BDVCM of bivariate monitoring variables can be obtained through combining the built BDLM about two monitoring variables and Gaussian copula function of bivariate monitoring variables. Further, the BDVCM of multiple monitoring variables can be obtained through connecting the BDVCM of any two monitoring variables with the vine structure. The detailed modeling processes are as follows.

With equation (6), it is known that two one-step prediction variables $\left(y_{1}, y_{2}\right)$ both follow normal distribution; namely,

$$
\begin{aligned}
& y_{1, t+1} \sim \mathrm{N}\left(\mu_{1, t+1}, \sigma_{1, t+1}^{2}\right), y_{2, t+1} \sim \mathrm{N}\left(\mu_{2, t+1}, \sigma_{2, t+1}^{2}\right), \\
& F_{1}\left(y_{1, t+1}\right)=\Phi\left(\frac{y_{1, t+1}-\mu_{1, t+1}}{\sigma_{1, t+1}}\right), \\
& F_{2}\left(y_{2, t+1}\right)=\Phi\left(\frac{y_{2, t+1}-\mu_{2, t+1}}{\sigma_{2, t+1}}\right) .
\end{aligned}
$$

Let

$$
\begin{aligned}
& u_{1}=F_{1}\left(y_{1, t+1}\right), \\
& u_{2}=F_{2}\left(y_{2, t+1}\right) .
\end{aligned}
$$

Further, BDVCM about bivariate monitoring variables can be obtained with

$$
\begin{aligned}
\left(\begin{array}{c}
y_{1, t+1}, \\
y_{2, t+1}
\end{array}\right) \sim C\left(\begin{array}{c}
F_{1}\left(y_{1, t+1}\right), \\
F_{2}\left(y_{2, t+1}\right)
\end{array}\right)=C\left(\begin{array}{c}
u_{1}, \\
u_{2} ; \rho_{t+1}
\end{array}\right) \\
=\Phi_{G}\left(\begin{array}{c}
F_{1}\left(y_{1, t+1}\right), \\
F_{2}\left(y_{2, t+1}\right) ; \rho_{t+1}
\end{array}\right),
\end{aligned}
$$




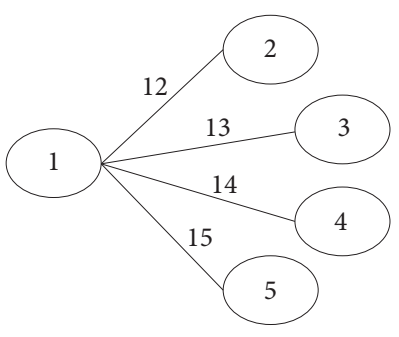

Tree 1

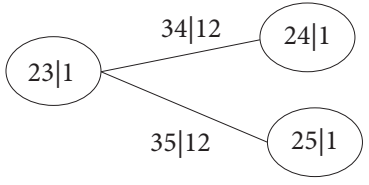

Tree 3

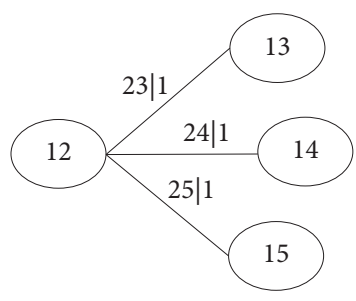

Tree 2

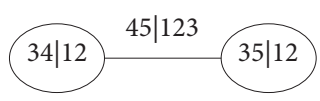

Tree 4

FIgURE 1: Five-dimensional C-vine structure.

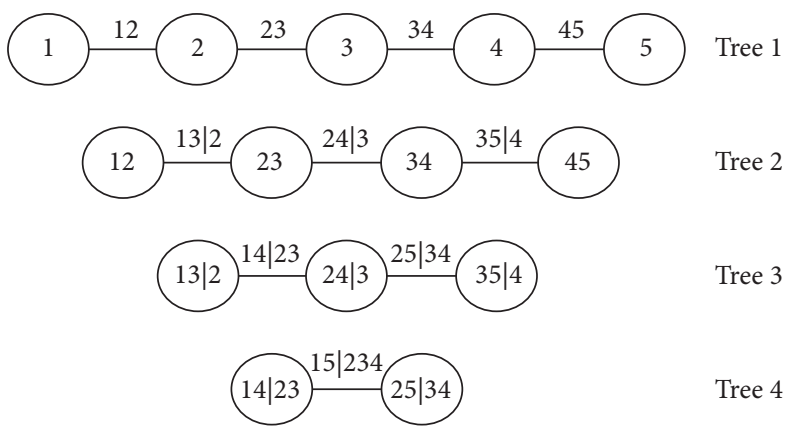

FIgURe 2: Five-dimensional D-vine structure.

where $\rho_{t}$ is the dynamic relevant parameter of bivariate Gaussian copula function, which can be computed with equation (17). $\Phi_{G}(\cdot)$ is Gaussian copula function.

With equation (20) and vine structures shown in Figures 1 and 2, the BDVCM of five-dimensional monitoring variables can be reached.

\section{Dynamic Reliability Prediction of the Steel Box Girder considering Nonlinear Correlation among Performance Functions about Failure Modes for Five Cross Sections Based on SHM Data}

With equations (16)-(20) and Section 2.3, the BDVCM, considering nonlinear dependence among the five monitoring points of five cross sections, is provided. Further, bridge girder system reliability can be dynamically predicted. Reliability indices and failure probability about multiple monitoring points can be solved with the FOSM method (see Section 3.1). Failure probability of bridge girder system can be computed through Section 3.2.

3.1. Reliability Prediction Formula Based on the FOSM Method. In this study, the adopted bridge girder has five monitoring cross sections, and each cross section has a control monitoring point [12]. The five predicted performance functions about the five control monitoring points are

$$
h_{i}\left(y_{i, t}\right)=[\sigma]-\gamma_{P} y_{i, t}, \quad i=1,2, \ldots, 5,
$$

where $h_{i}$ is the $i^{\text {th }}$ performance function, $[\sigma]$ is the strength (allowable stress), $y_{i, t}$ is the monitoring or predicted everyday extreme stress of the $i^{\text {th }}$ control monitoring point at time $t$, and $\gamma_{P}=1.15$ is the correction factor of the sensors [7].

Based on the FOSM method [13], the predicted reliability index $\beta_{i, t}$ and the corresponding failure probability $p_{f_{i, t}}$ of the $i^{\text {th }}$ control monitoring point can be solved with

$$
\begin{aligned}
\beta_{i, t} & =\frac{\mu_{[\sigma]}-\gamma_{P} \mu_{y_{i, t}}}{\sqrt{\sigma_{[\sigma]}^{2}+\left(\gamma_{P} \sigma_{y_{i, t}}\right)^{2}}} \\
p_{f_{i, t}} & =\Phi\left(-\beta_{i, t}\right) .
\end{aligned}
$$

3.2. The Steel Box Girder Reliability Prediction Based on $B D V C M$. This section supposes that any system composed of two control monitoring points is parallel. With equation (21), the failure probability of parallel system composed of two control monitoring points can be computed with the following equation: 


$$
\begin{aligned}
g_{i, t+1} & =[\sigma]-\gamma_{P} y_{i, t+1}, \quad i=1,2, \\
P\left(g_{1, t+1} \leq 0, g_{2, t+1} \leq 0 ;\right) & =P\left(G_{1}\left(g_{1, t+1}\right) \leq G_{1}(0), G_{2}\left(g_{2, t+1}\right) \leq G_{2}(0)\right) \\
& =P\left(F_{1, t+1} \leq G_{1}(0), F_{2, t+1} \leq G_{2}(0)\right) \\
& =C\left(G_{1}(0), G_{2}(0) ; \rho_{12, t+1}\right)=C\left(p_{f_{1, t+1}}, p_{f_{2, t+1}} ; \rho_{t+1}\right) \\
& =\Phi_{G}\left(p_{f_{1, t+1}}, p_{f_{2, t+1}} ; \rho_{t+1}\right) .
\end{aligned}
$$

where $\quad F_{i, t+1}=\Phi\left([\sigma]-\gamma_{P} y_{i, t+1}-\gamma_{P} \mu_{i, t+1} / \gamma_{P} \sigma_{i, t+1}\right), G_{i}(0)=\Phi$ $\left(-\mu_{i, t+1} / \sigma_{i, t+1}\right), i=1,2, p_{f_{1, t+1}}$ and $p_{f_{2, t+1}}$ can be computed with equation (23). $C$ is copula function, and $\rho_{t+1}$ is the time-variant relevant parameter obtained with equation (17). $\Phi_{G}(\cdot)$ is Gaussian copula function.
Because the multiple parallel systems composed of any two control monitoring points are serial, with equations (21)-(24), the predicted failure probability of the steel box girder considering the nonlinear correlation among performance functions of failure modes about multiple control monitoring points can be solved with the following equation:

$$
P_{f_{\text {system }, t+1}}=\max \left\{\begin{array}{c}
C\left(p_{f_{1, t+1}}, p_{f_{2, t+1}} ; \rho_{12, t+1}\right), C\left(p_{f_{1, t+1}}, p_{f_{3, t+1}} ; \rho_{13, t+1}\right), C\left(p_{f_{1, t+1}}, p_{f_{4, t+1}} ; \rho_{14, t+1}\right), C\left(p_{f_{1, t+1}}, p_{f_{5, t+1}} ; \rho_{15, t+1}\right), \\
C\left(p_{f_{2, t+1}}, p_{f_{3, t+1}} ; \rho_{23, t+1}\right), C\left(p_{f_{2, t+1}}, p_{f_{4, t+1}} ; \rho_{24, t+1}\right), C\left(p_{f_{2, t+1}}, p_{f_{5, t+1}} ; \rho_{25, t+1}\right), \\
C\left(p_{f_{3, t+1}}, p_{f_{4, t+1}} ; \rho_{34, t+1}\right), C\left(p_{f_{3, t+1}}, p_{f_{5, t+1}} ; \rho_{35, t+1}\right), \\
C\left(p_{f_{4, t+1}}, p_{f_{5, t+1}} ; \rho_{45, t+1}\right)
\end{array}\right\} .
$$

\section{Application to an Existing Bridge}

This paper takes Tianjin Fumin Bridge from China as the analytical example $[7,14,15]$. The adopted girder has five monitoring cross sections which are sections A, B, C, D, and E shown in Figure 3. Each cross section has installed three stress sensors at three different monitoring points which are all Fiber Bragg Grating sensors [12]. The longitudinal stresses about these five sections are monitored through an array of stress sensors [12]. These stress sensors about the five monitoring cross sections are shown in Table 1 and Figure 4, and these cross sections are serial. The steel type of the steel box girder is $\mathrm{Q} 345 \mathrm{qD}$, and the mean value and coefficient of variance are, respectively, $411 \mathrm{MPa}$ and 0.098 [12].

4.1. Monitoring Everyday Extreme Stress Data of the Five Monitoring Cross Sections. Sampling rate about the stress data is $2.5 \mathrm{~Hz}$ sampling frequency. The everyday extreme stresses of the five cross sections are, respectively, monitored for two hundred days. The monitoring data are shown in Figure 5, which show that each section is monitored by three sensors at three different locations and the monitoring data are dynamic and random [12]. According to measurement program [7, 15], these data can reflect the safety-based dynamic reliability of girder system. The maximum absolute value of everyday extreme stresses at the three points of each section is defined as the everyday extreme stress of each section [12].
4.2. BDLM of Monitoring Everyday Extreme Stress Data of Each Monitoring Section. Figure 5 shows that the sensors FBG01074, FBG01947, FBG01071, FBG01015, and FBG01028, respectively, monitored the everyday extreme stress about the control locations of sections A, B, C, D, and E. So, the monitoring data of the five sensors are applied to build the BDLM for predicting the corresponding everyday extreme stresses. The detailed contents are as follows.

Firstly, the monitoring extreme data of the five sensors are, respectively, smoothly processed with cubical smoothing algorithm with five-point approximation [7], and the processed data can be approximately considered as the initial state information [12]. Through Kolmogorov-Smirnov (K-S) test, these initial state data about the five sensors are all approximately simulated with normal distribution. Secondly, based on equations (1)-(9) of Section 2.1 including Sections 2.1.1-2.1.3, the BDLM can be easily built. Finally, with the built BDLM, the everyday extreme data can be dynamically predicted. The predicted results are shown in Figure 6. As can be seen, the predicted extreme stress data and the predicted extreme stress ranges all fit the changing rules of monitoring extreme stress data.

\subsection{Dynamic Reliability Prediction of the Bridge Girder System}

4.3.1. Bridge Girder System Reliability Prediction without considering the Dependence among the Multiple Failure Modes. In this section, dynamic reliability index data at the control points in Tianjin Fumin Bridge girder system are 


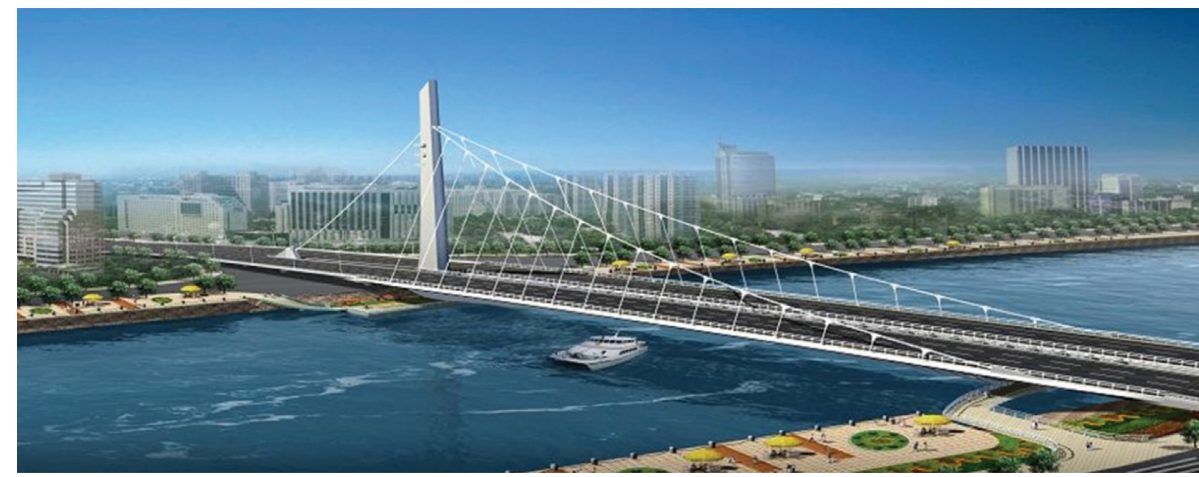

(a)

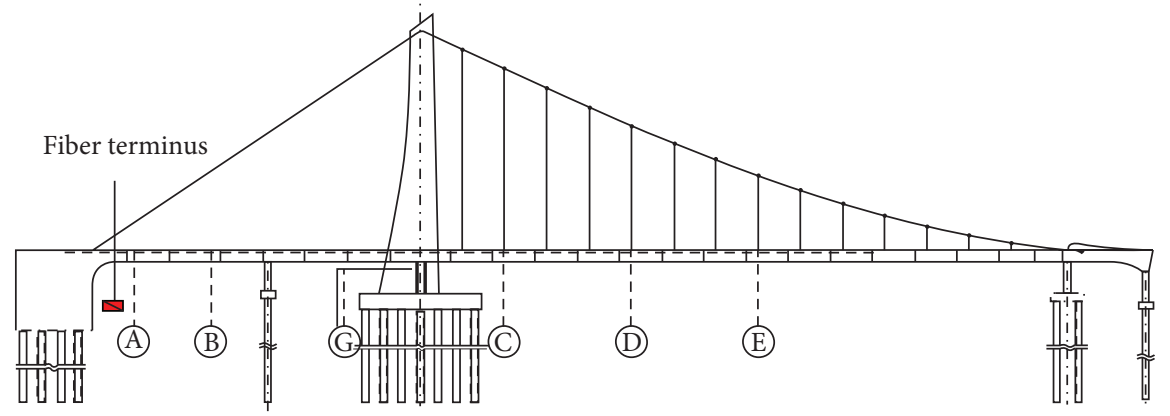

(b)

Figure 3: (a) Fumin Bridge. (b) Five monitoring sections of Fumin Bridge girder system.

TABLE 1: Stress sensors of the five monitoring cross sections [12].

\begin{tabular}{lc}
\hline Sections & Different stress sensors at different monitoring points \\
\hline A & FBG01074, FBG01081, FBG01078 \\
B & FBG01947, FBG01949, FBG01046 \\
C & FBG01059, FBG01071, FBG01073 \\
D & FBG01012, FBG01015, FBG01005 \\
E & FBG01029, FBG01021, FBG01028 \\
\hline
\end{tabular}

predicted with equation (22) and the built BDLM. The allowable stress of bridge steel follows normal distribution with $411 \mathrm{MPa}$ (mean value) and 0.098 (coefficient of variance).

With equation (22), the computation formula of reliability indices can be obtained with

$$
\begin{aligned}
& \beta_{i, P, t}=\frac{411-1.15 \mu_{i, t}}{\sqrt{(411 \times 0.098)^{2}+\left(1.15 \sigma_{i, t}\right)^{2}}}, i=1,2, \ldots, 5, \\
& p_{f_{i, P, t}}=\Phi\left(-\beta_{i, P, t}\right), \quad i=1,2, \ldots, 5,
\end{aligned}
$$

where $\beta_{i, P, t}$ is the predicted reliability index at the $i^{\text {th }}$ monitoring point at time $t ;\left(\mu_{i, t}, \sigma_{i, t}\right)$ can be obtained with equation (6).

With equation (26), the reliability indices of the five control monitored points can be dynamically predicted, and the predicted results are shown in Figure 7, from which, as can be seen, the predicted reliability indices and the predicted ranges all fit the changing rules of monitored reliability indices.
The comparative analysis is made, and the minimum value is selected as bridge girder reliability index. The results are shown in Figure 8, from which, as can be noticed, the reliability indices of the control monitored point at section $B$ are the bridge girder reliability indices.

Based on these solved reliability indices and equation (27), failure probability of the bridge girder system can be calculated. The comparative analysis about the failure probability of the five control points is made, and the maximum value shown in Figure 9 is selected as the time-variant failure probability of the steel box girder. From Figure 9, as can be seen, the failure probability of the control monitoring point at section $\mathrm{B}$ is the bridge girder failure probability.

\subsubsection{Bridge Girder Reliability Prediction considering the} Dependence among the Multiple Failure Modes. In this section, dynamic reliability of steel box girder considering correlation is predicted. Based on C-vine structure shown in Figure 1, with equation (16), the time-variant correlation coefficients (e.g., $\rho(A B), \rho(A C), \rho(A D), \rho(A E), \quad \rho(B C \mid A)$, $\rho(B D \mid A), \rho(B E \mid A), \rho(C D \mid A B), \quad \rho(C E \mid A B), \rho(D E \mid A B C))$ between the performance functions of any two points can be obtained, where the time-variant correlation coefficients are equal to the relevant parameters of the corresponding copula functions. $\rho(A B), \rho(A C), \rho(A D), \rho(A E)$, and $\rho(B C \mid A)$ are shown in Figure 10(a), and $\rho(B D \mid A), \rho(B E \mid A), \rho(C D \mid A B)$, $\rho(C E \mid A B)$, and $\rho(D E \mid A B C)$ are displayed in Figure 10(b). 


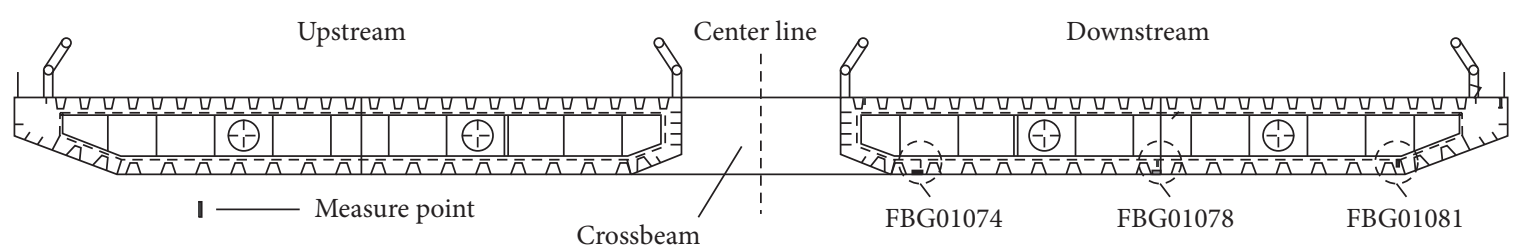

(a)

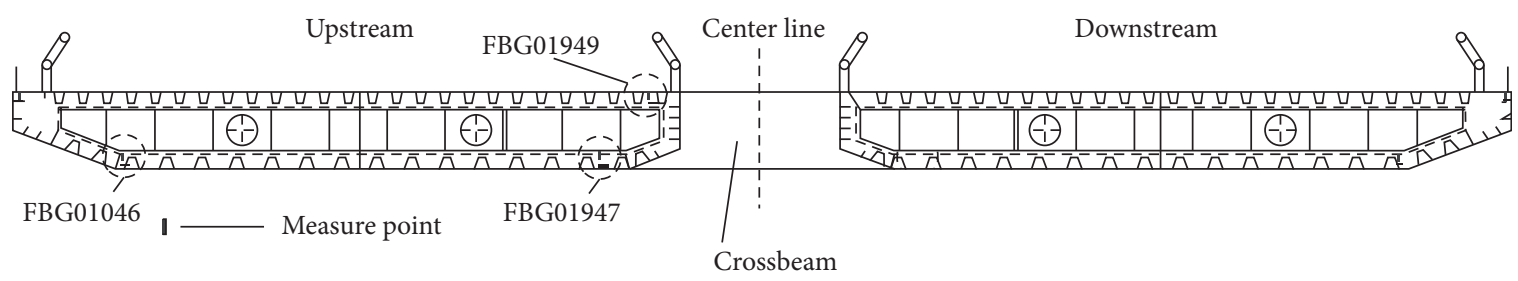

(b)

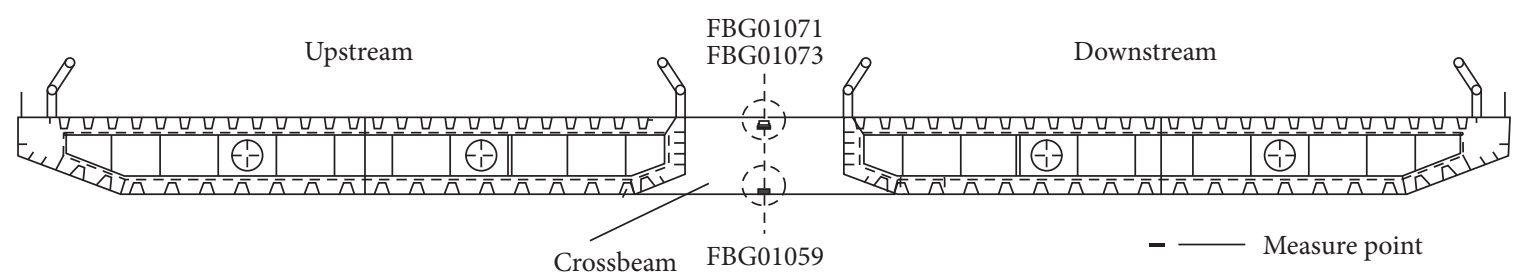

(c)

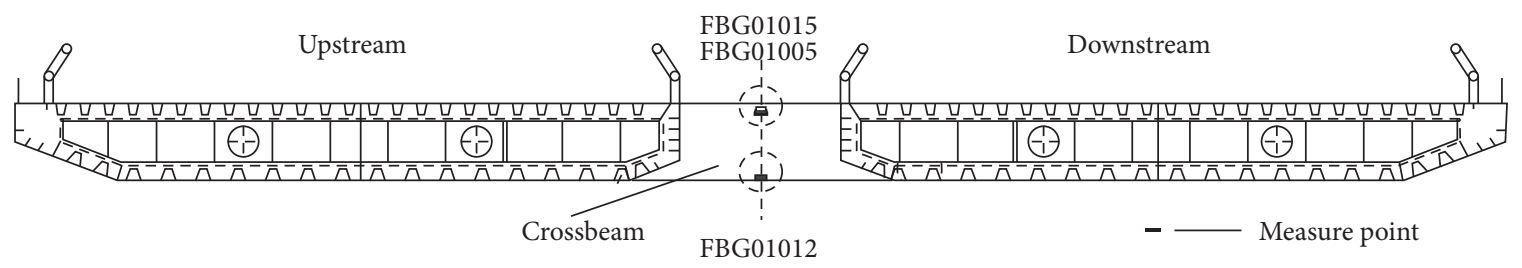

(d)

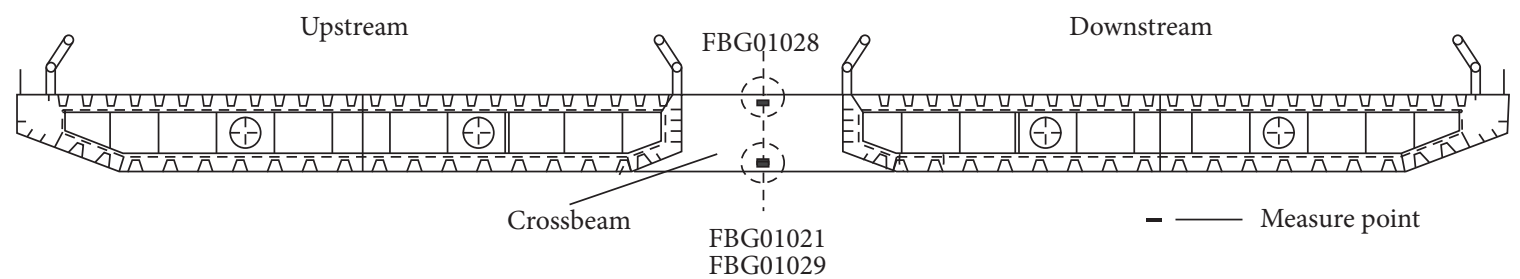

(e)

Figure 4: Stress sensors of the five monitoring cross sections. (a) Section A. (b) Section B. (c) Section C. (d) Section D. (e) Section E.

Based on Figure 10, bivariant Gaussian-copula PDF functions and contour maps considering nonlinear dependence between any pair of failure modes about any pair of control monitoring points are analyzed. Conditional on sections $\mathrm{A}$ and $\mathrm{B}$, the time-variant correlation between sections $\mathrm{C}$ and $\mathrm{D}$ was analyzed, and the analytical results of the $100^{\text {th }}$ day are selected and shown in Figure 11, from which, as can be known, the dependence between any pair of failure modes is nonlinear.
With equation (24), $p_{f}(A B), p_{f}(A C), p_{f}(A D)$, $p_{f}(A E)$, and $p_{f}(B C \mid A)$ can be computed and shown in Figure $12(\mathrm{a})$, and $p_{f}(B D \mid A), p_{f}(B E \mid A), p_{f}(C D \mid A B)$, $p_{f}(C E \mid A B)$, and $p_{f}(D E \mid A B C)$ can be solved and shown in Figure 12(b). Further, with equation (24), girder system failure probability considering nonlinear dependence among the failure modes can be obtained and shown in Figure 13, from which, as can be known, the bridge girder system failure probability taking into account the nonlinear dependence among the performance 

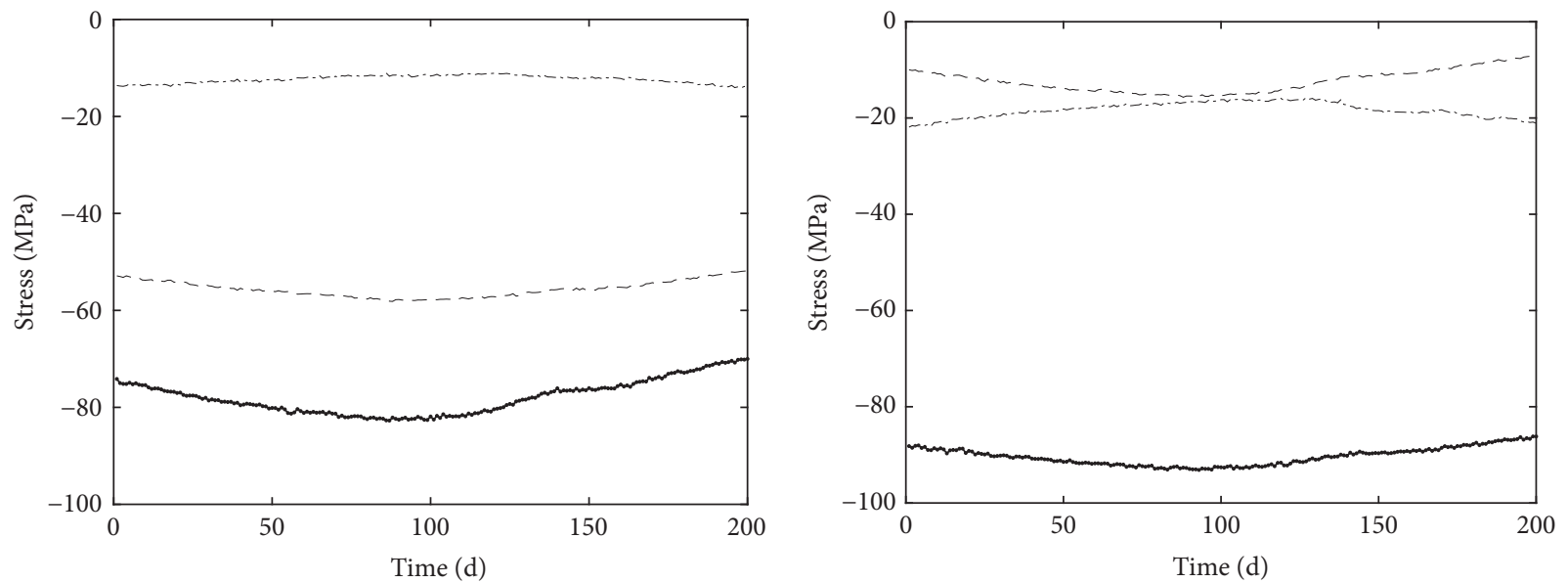

- FBG01074

- FBG01947

- - FBG01949

- - - FBG01046

(a)

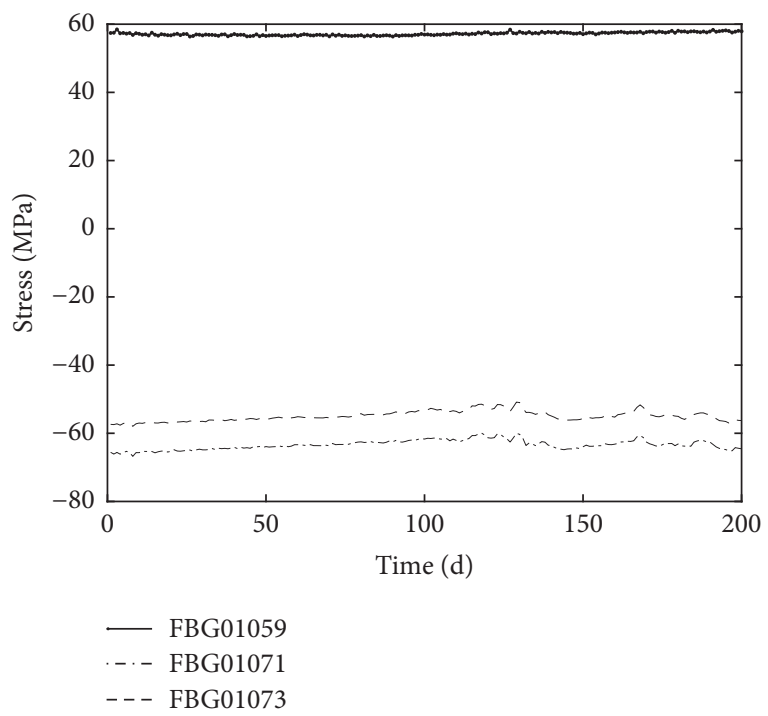

(c)

(d)

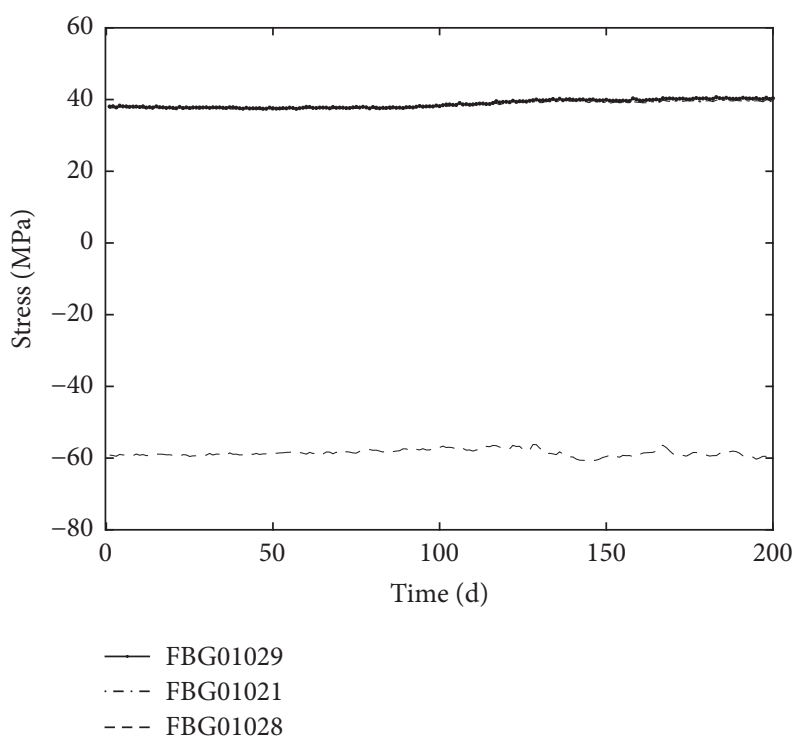

(e)

FIgUre 5: Monitoring extreme stresses of the five sections. (a) Section A. (b) Section B. (c) Section C. (d) Section D. (e) Section E. 


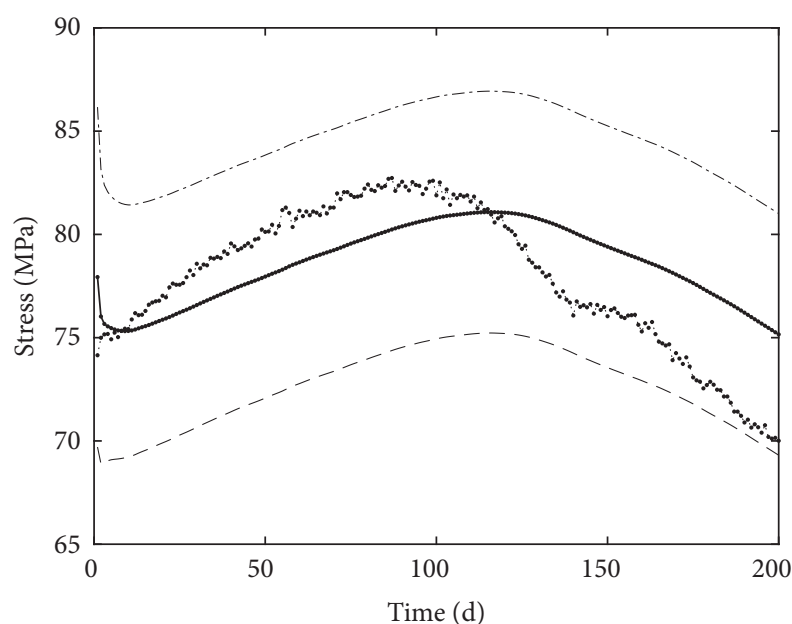

...... Monitoring extreme data $\quad$...- Predicted upper data - Predicted extreme data

(a)

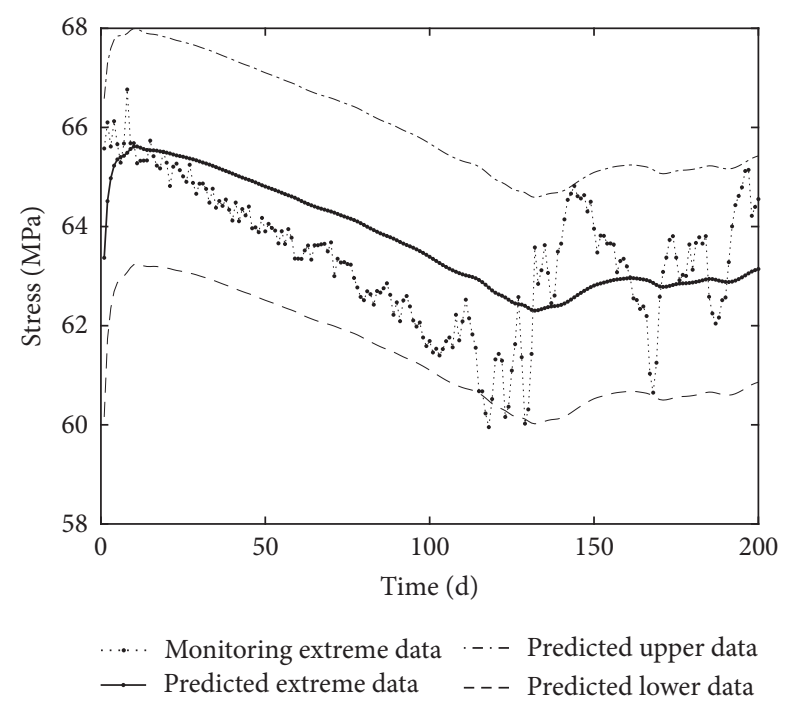

(c)

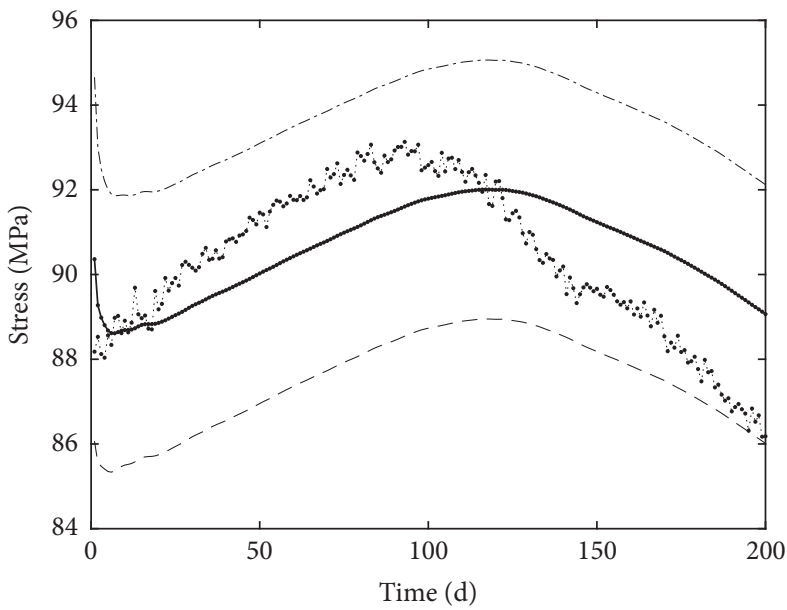

..... Monitoring extreme data - - - Predicted upper data

Predicted extreme data $\quad$ - - Predicted lower data

(b)

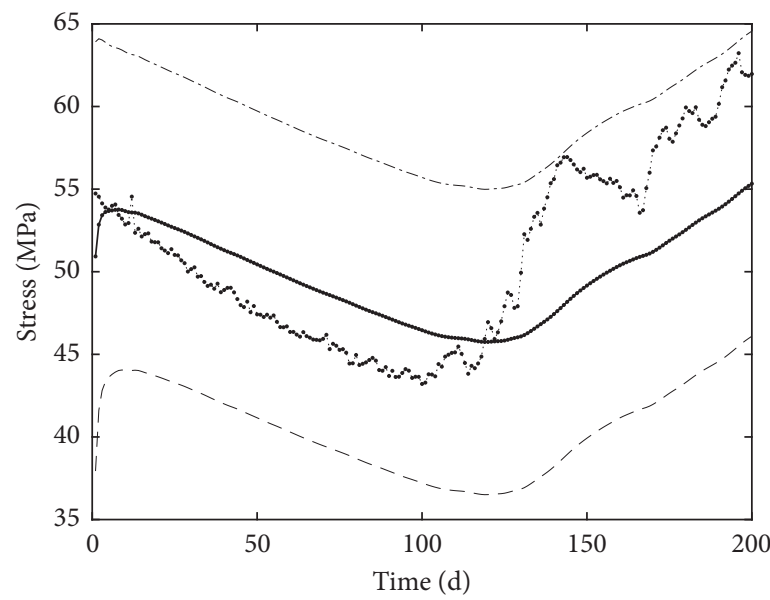

..... Monitoring extreme data -..- Predicted upper data Predicted extreme data - - - Predicted lower data

(d)

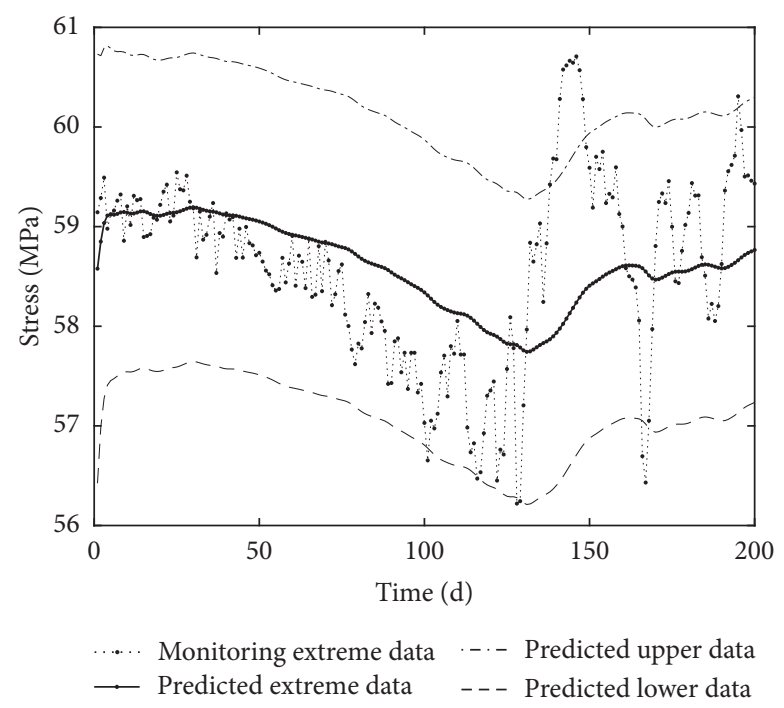

(e)

Figure 6: Predicted extreme stresses of the five sections. (a) Section A. (b) Section B. (c) Section C. (d) Section D. (e) Section E. 

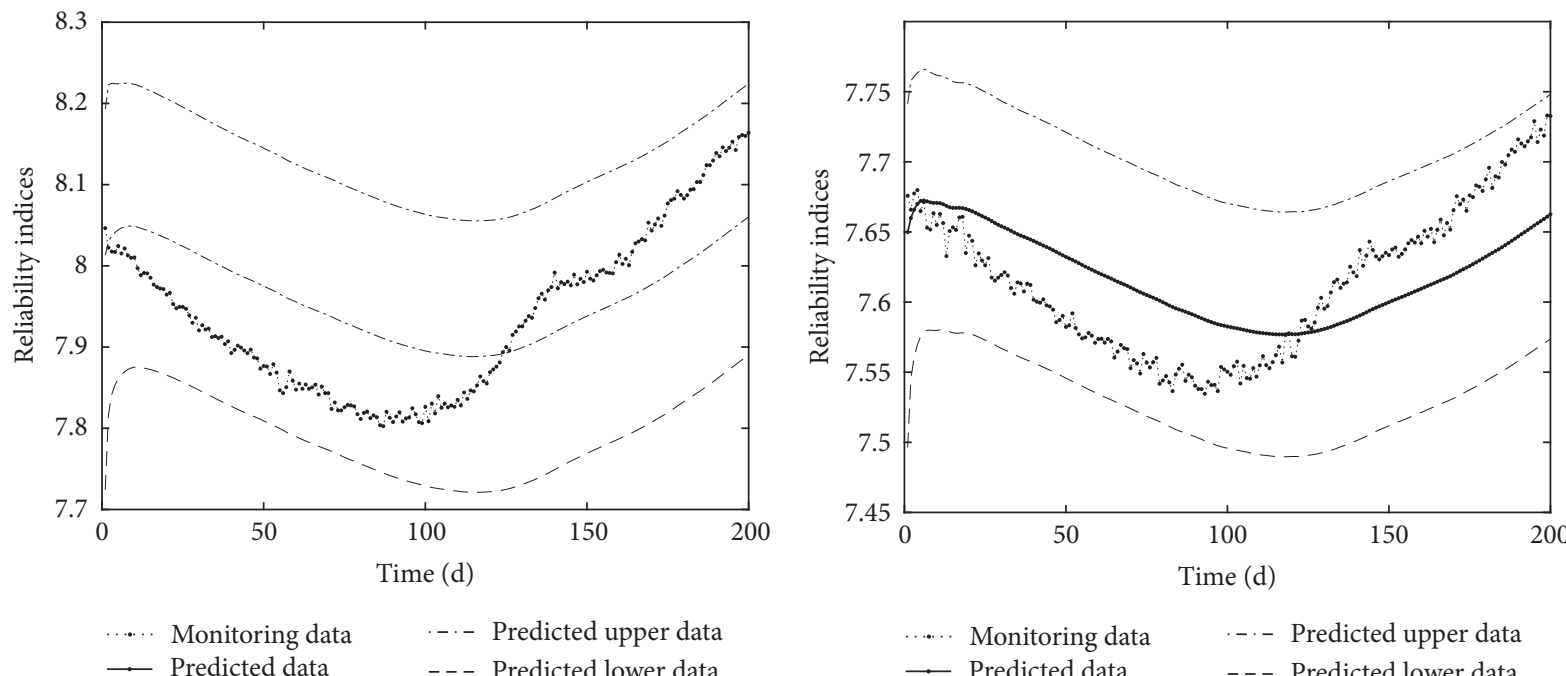

..... Monitoring data -..- Predicted upper data

a)

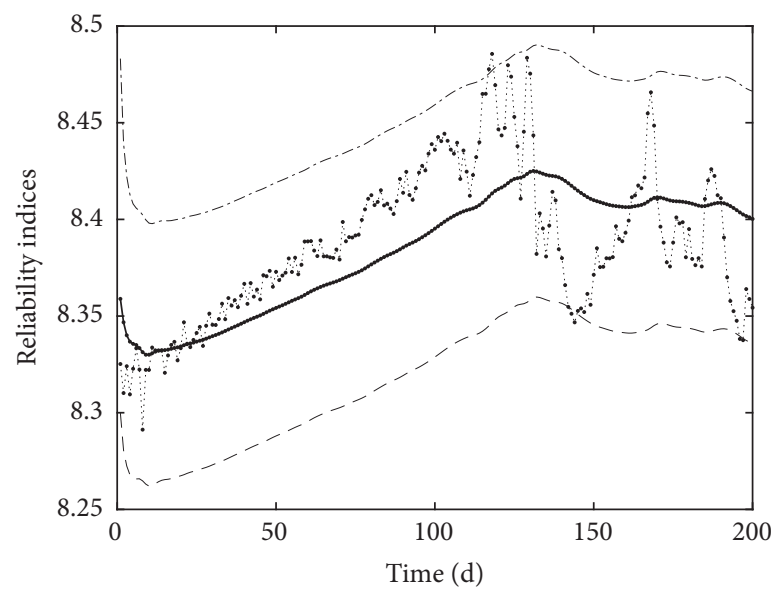

(b)
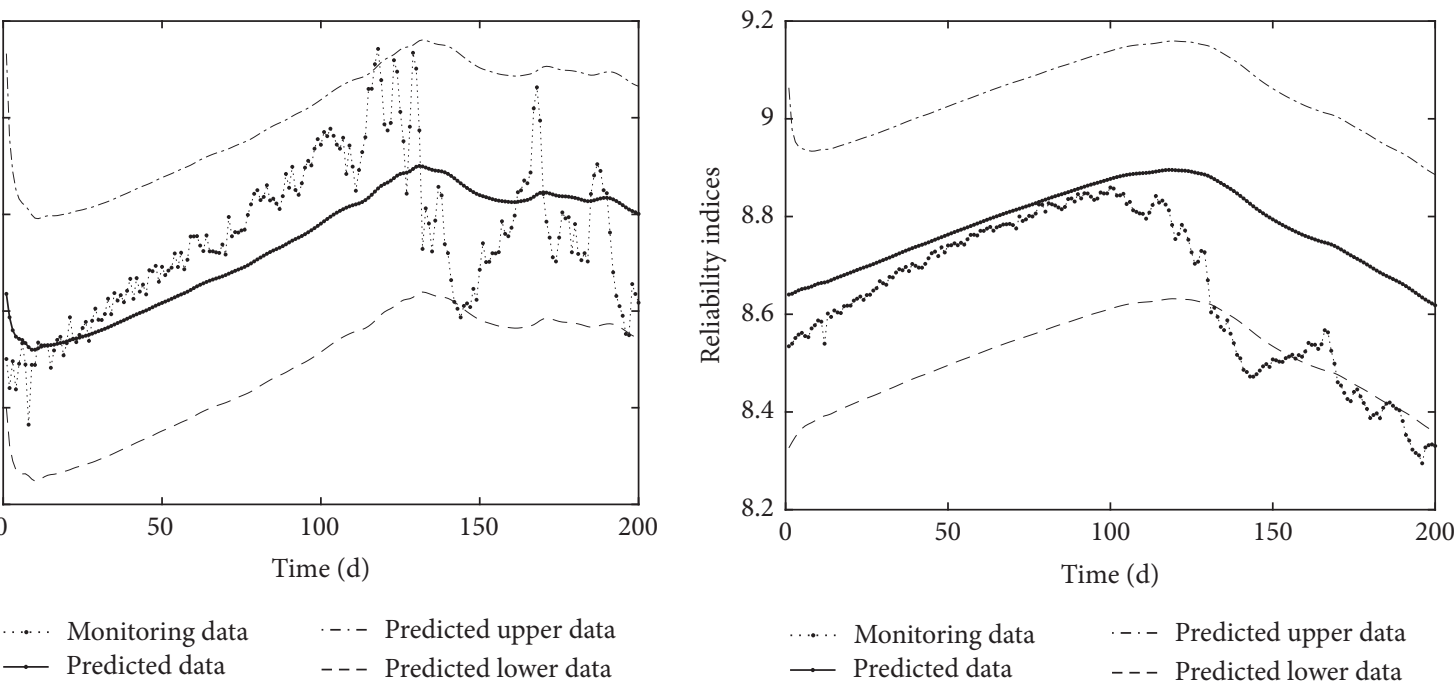

$\begin{array}{lll}\ldots . . . & \text { Monitoring data } \\ \text {-. } & \text { Predicted data } & \text { - - - Predicted upper data }\end{array}$

(d)

(c)

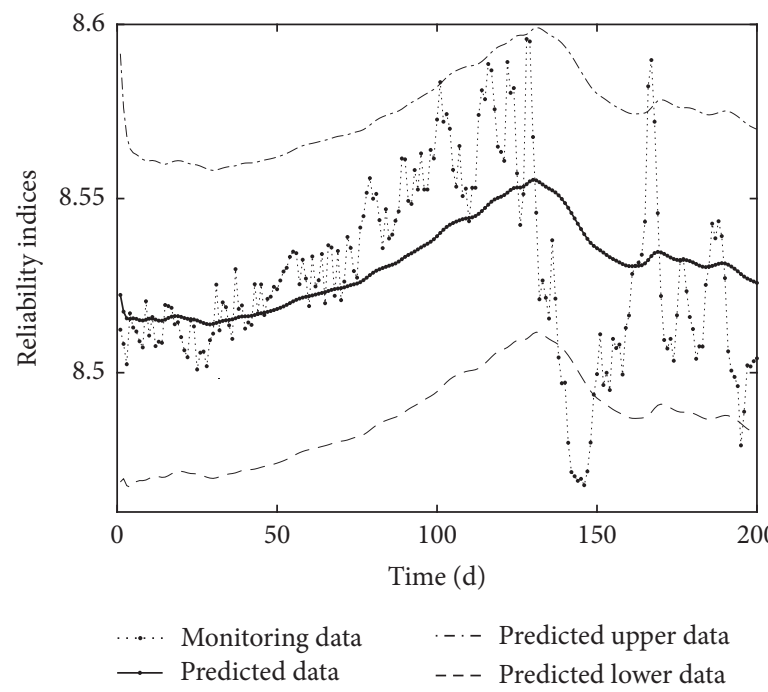

(e)

Figure 7: Predicted reliability indices of the five sections. (a) Section A. (b) Section B. (c) Section C. (d) Section D. (e) Section E. 


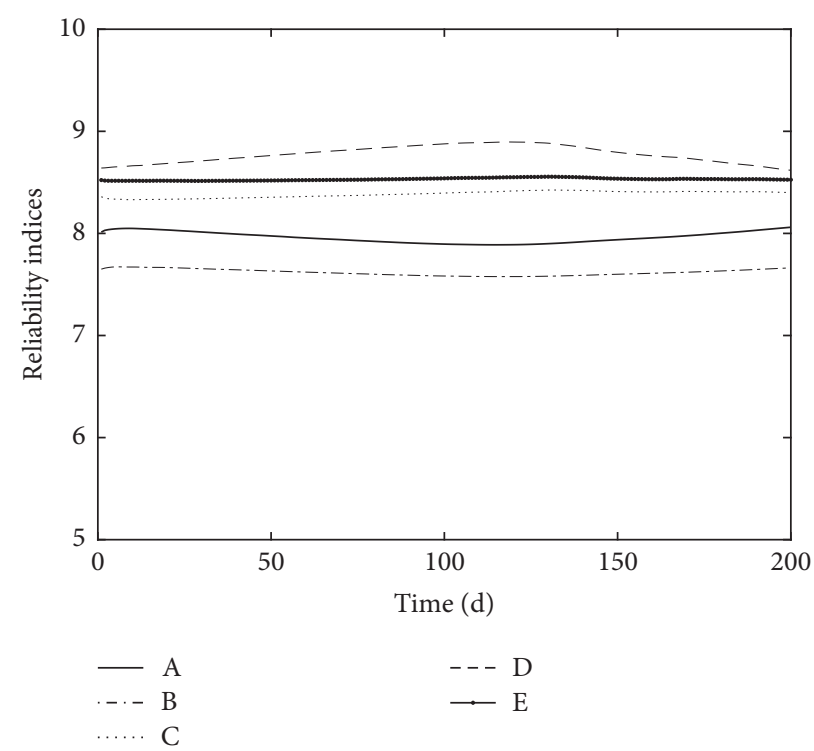

FIGURE 8: Comparative analysis of predicted reliability indices.

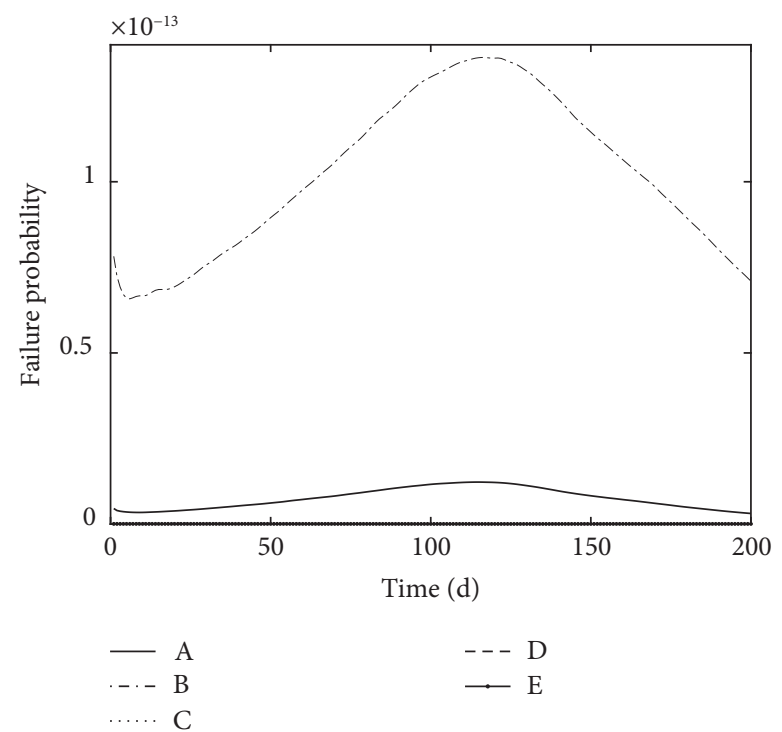

FIGURE 9: Comparative analysis of predicted failure probability.

functions of failure modes is smaller than the one obtained without taking into account the nonlinear dependence, which proves that it is more reasonable and necessary to take into account the nonlinear dependence among the performance functions of failure modes for dynamic reliability prediction of structural system. 


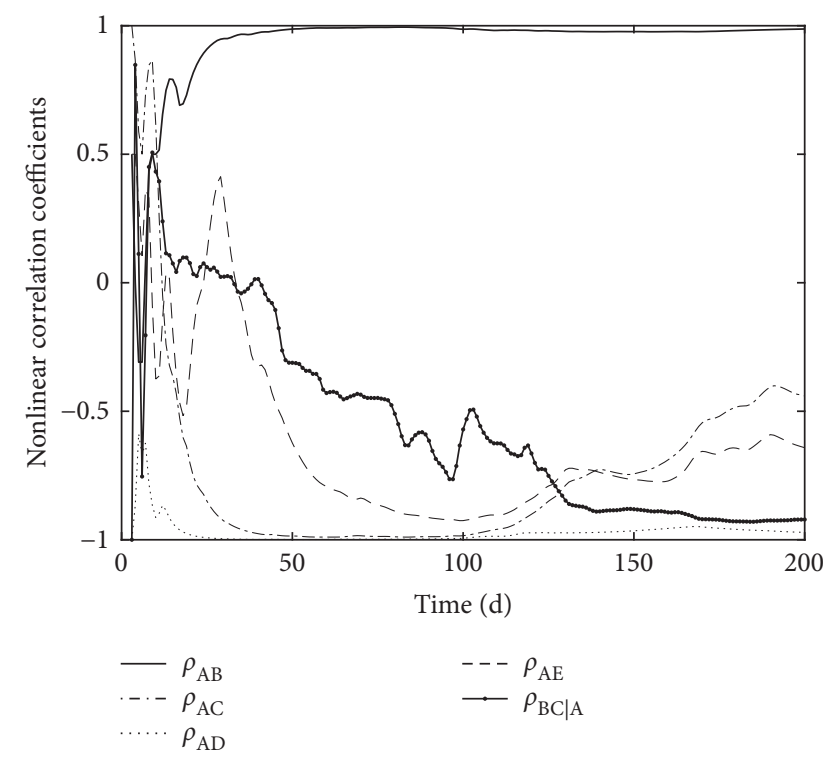

(a)

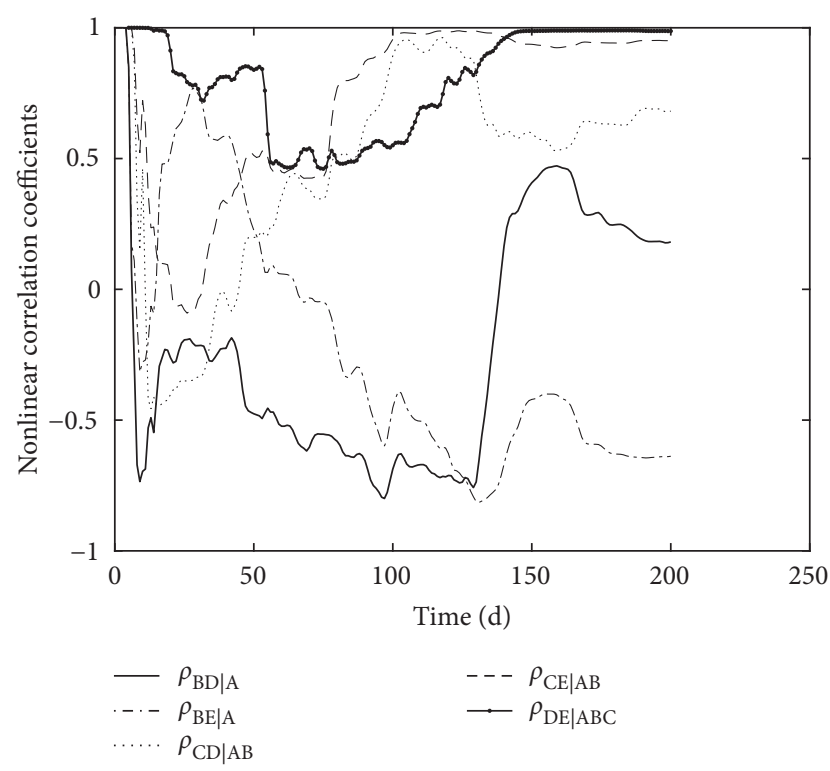

(b)

FIGURE 10: Time-dependent nonlinear correlation coefficients.
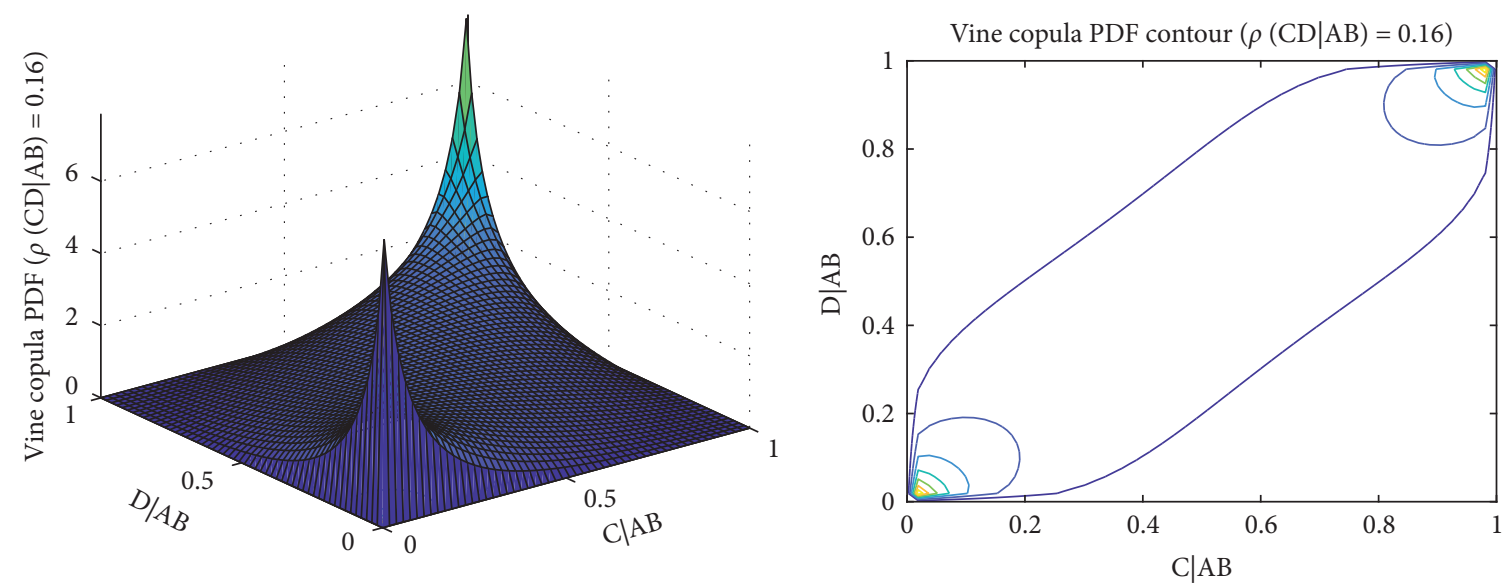

The $100^{\text {th }}$ day

Figure 11: The time-variant correlation between sections $\mathrm{C}$ and $\mathrm{D}$ conditional on sections $\mathrm{A}$ and $\mathrm{B}$. 


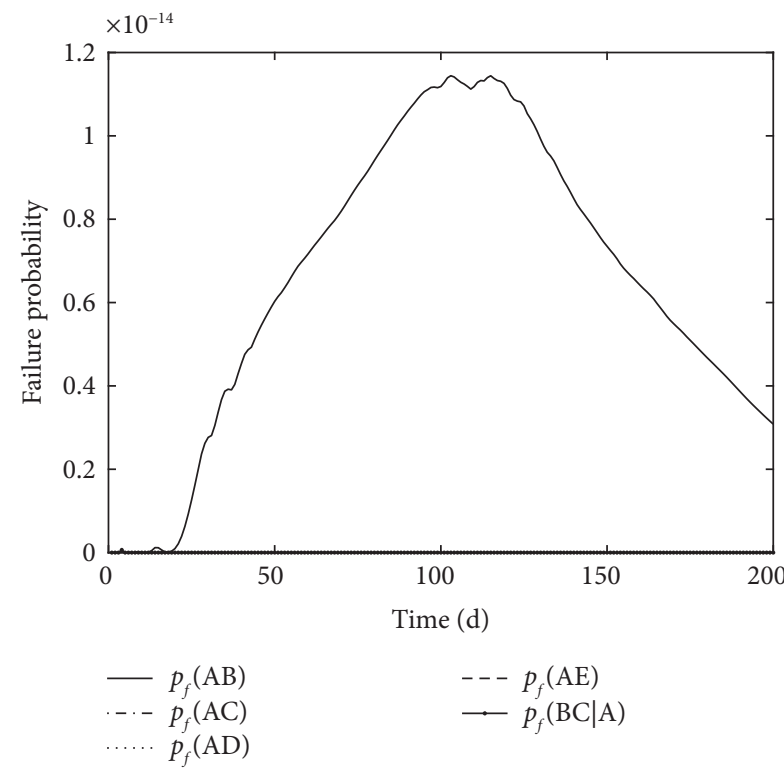

(a)

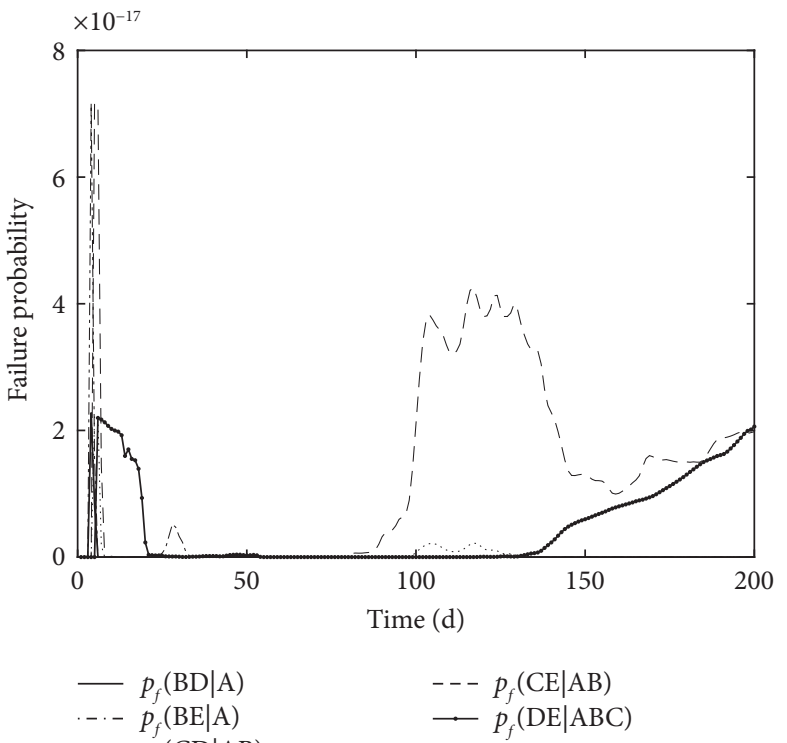

(b)

FIGURE 12: Failure probability of bivariate vine structure.

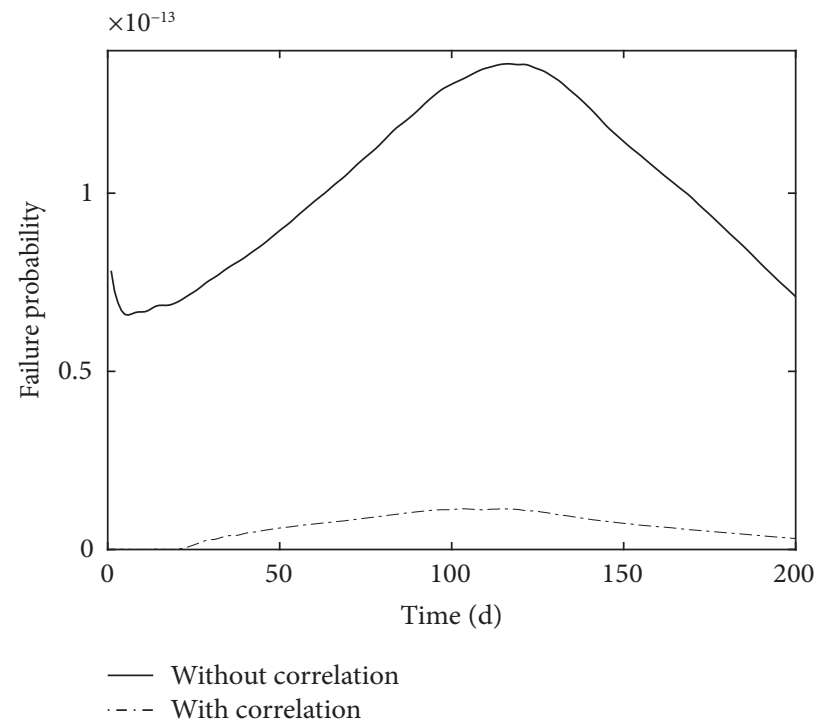

FIGURE 13: Predicted bridge girder failure probability.

\section{Conclusions}

This paper presented a new dynamic reliability prediction method for the steel box girder. By combining Bayesian Dynamic Linear Model (BDLM) with vine copula functions, the Bayesian Dynamic Vine Copula Model (BDVCM) is provided. The predicted results illustrate that the dynamic failure probability taking into account the nonlinear dependence is lower than the one without taking into account the nonlinear dependence. It is indicated that the computed results without taking into account the dynamic nonlinear dependence are conservative, and the predicted results taking into account the dynamic nonlinear dependence are more reasonable and necessary.

\section{Data Availability}

Some or all data, models, or codes generated or used during the study are available from the corresponding author upon request (list items). 


\section{Conflicts of Interest}

The authors declare no conflicts of interest regarding the publication of this article.

\section{Acknowledgments}

The authors disclosed receipt of the following financial support for the research, authorship, and/or publication of this article: This work was supported by Natural Science Foundation of Gansu Province of China (20JR10RA625, 20JR10RA623), National Key Research and Development Project of China (Project No. 2019YFC1511005), Fundamental Research Funds for the Central Universities (Grant No. lzujbky-2020-55) and National Natural Science Foundation of China (Grant No. 51608243).

\section{References}

[1] Á. Rózsás and Z. Mogyorósi, "The effect of copulas on time-variant reliability involving time-continuous stochastic processes," Structural Safety, vol. 66, p. 94, 2017.

[2] X.-S. Tang, D.-Q. Li, C.-B. Zhou, K.-K. Phoon, and L.-M. Zhang, "Impact of copulas for modeling bivariate distributions on system reliability," Structural Safety, vol. 44, p. 80, 2013.

[3] X. P. Fan and Y. F. Liu, "Time-variant reliability prediction of bridge system based on BDGCM and SHM data," Structural Control and Health Monitoring, vol. 25, no. 7, p. e2185, 2018.

[4] Y. F. Liu, X. P. Fan, and D. G. Lu, "Time-dependent uncertainty analysis of structures based on copula functions," Vulnerability, Uncertainty, and Risk (CASCE, Liverpool, Liverpool, UK, 2014.

[5] Y. F. Liu and X. P. Fan, "Gaussian Copula-Bayesian dynamic linear model-based time-dependent reliability prediction of bridge structures considering nonlinear correlation between failure modes," Advances in Mechanical Engineering, vol. 8, no. 11, pp. 1-15, 2016.

[6] Y. F. Liu, System reliability analysis of bridge structures considering correlation of failure modes and proof modes, Thesis (PhD), Harbin Institute of Technology, Harbin, China, 2015.

[7] X. P. Fan, Reliability Updating and Bayesian Prediction of Bridges Based on Proof Loads and Monitoring data, Thesis (PhD), Harbin Institute of Technology, Harbin, China, 2014.

[8] T. Bedford and R. M. Cooke, "Probability density decomposition for conditionally dependent random variables modeled by vines," Annals of Mathematics \& Artificial Intelligence, vol. 32, no. 1-4, pp. 245-268, 2001.

[9] T. Bedford and R. M. Cooke, "Vines--a new graphical model for dependent random variables," The Annals of Statistics, vol. 30, no. 4, pp. 1031-1068, 2002.

[10] A. H.-S. Ang and W. H. Tang, Probability concepts in engineering planning and design, John Wiley \& Sons, NewYork, 1975.

[11] R. B. Nelsen, An Introduction to Copulas, Springer Series in Statistics, Berlin, Germany, 2006.

[12] Y. F. Liu and X. P. Fan, "Dynamic reliability prediction for the steel box girder based on MBDGCM and SHM extreme stress data," Structural Control and Health Monitoring, vol. 27, no. 6, pp. 1-17, 2020.

[13] R. E. Melchers, Structural Reliability, Analysis and Prediction, Ellis Horwood, Chichester, UK, 1987.
[14] Y. Sun, H.-P. Zhu, and D. Xu, "A specific rod model based efficient analysis and design of hanger installation for self-anchored suspension bridges with 3D curved cables," Engineering Structures, vol. 110, pp. 184-208, 2016.

[15] S. F. ChenD. W. Yang et al., As-built Drawings and Measurement Programs of Fumin Bridge Health Monitoring Project, Harbin Institute of Technology, Harbin, China, 2010. 